\title{
ОСОБЕННОСТИ ФУНКЦИОНАЛЬНОГО СОСТОЯНИЯ ПАЦИЕНТОВ С ОСТРОЙ КОРОНАРНОЙ ПАТОЛОГИЕЙ, СВЯЗАННЫЕ С ДОСТИЖЕНИЕМ ЦЕЛЕВЫХ ЗНАЧЕНИЙ ЧСС
}

\author{
() Михин В.П. ${ }^{1}$, Коробова В.Н. ${ }^{1}$, Харченко А.В. ${ }^{1}$, Чернятина М.А. ${ }^{1}$, Громнацкий Н.И. ${ }^{1}$, Спасский А.А. ${ }^{2}$, \\ Каюшников А.Б. ${ }^{2}$, Ледовский С.М. ${ }^{2}$, Маркина Т.Н. ${ }^{2}$
}

${ }^{1}$ Кафедра внутренних болезней № 2 Курского государственного медицинского университета, Курск;
${ }^{2}$ Национальное агентство клинической фармакологии и фармации, Москва E-mail: viktoria.korobova@mail.ru

Снижение летальности пациентов с острой коронарной патологией продолжает оставаться ведущей задачей кардиологии. ЧСС является одним из важных критериев адекватности проводимого лечения и реабилитации пациентов, поскольку достижение целевых значений сопровождается снижением относительного риска сердечной смерти и риска внезапной смерти. Проведенное исследование включало 86 пациентов с нестабильной стенокардией и 111 пациентов с острым инфарктом миокарда в возрасте 40-65 лет, которым проводилась оценка функционального состояния по интегральным показателям программно-аппаратного комплекса «Омега-М» на 3-и, 8-е и 14-е сут. Была установлена взаимосвязь ЧСС с параметром вегетативной регуляции, который может использоваться в качестве прогностического критерия достижения целевых значений ЧСС у пациентов с острой коронарной патологией на этапах госпитальной реабилитации.

Ключевые слова: частота сердечных сокращений, функциональное состояние, нестабильная стенокардия, острый инфаркт миокарда, программно-аппаратный комплекс «Омега-М».

\section{FEATURES OF FUNCTIONAL STATE ASSOCIATED WITH THE ACHIEVEMENT OF TARGET VALUES OF HEART RATE IN PATIENTS WITH ACUTE CORONARY PATHOLOGY Mikhin V.P. ${ }^{l}$, Korobova V.N. ${ }^{l}$, Kharchenko A.V. ${ }^{l}$, Chernyatina M.A. ${ }^{l}$, Gromnatskiy N.I. ${ }^{l}$, Spasskiy A.A. ${ }^{2}$, Kayushnikov A.B. ${ }^{2}$, Ledovskiy S.M. ${ }^{2}$, Markina T.N. ${ }^{2}$ \\ ${ }^{1}$ Department of Internal Medicine $N 2$ of Kursk State Medical University, Kursk; \\ ${ }^{2}$ National Agency of Clinical Pharmacology and Pharmacy, Moscow}

Reduction of mortality in patients with acute coronary pathology continues to be the leading task of cardiology. Heart rate is one of the important criteria for the adequacy of ongoing treatment and rehabilitation of patients, since the achievement of target values is accompanied by a decrease in the relative risk of cardiac death and the risk of sudden death. The study included 86 patients with unstable angina and 111 patients with acute myocardial infarction at the age of 40-65 years, whose functional state was evaluated by the Omega-M software-hardware complex according to the integrated parameters on the 3rd, 8th and 14th days. The correlation of heart rate with the parameter of vegetative regulation was discovered, that can be used as a prognostic criterion for achievement of target heart rate in patients with acute coronary pathology at stages of hospital rehabilitation.

Keywords: heart rate, functional state, unstable angina, acute myocardial infarction, software and hardware complex "Omega-M".

По прогнозам ВОЗ, к 2020 г. ишемическая болезнь сердца (ИБС) займет первое место среди всех причин инвалидизации и смертности в мире (WHO, 2003). Наиболее жизнеугрожающими нозологиями в структуре ИБС являются нестабильная стенокардия (НC) и острый инфаркт миокарда (ОИМ), которые ассоциируются с высоким риском нарушений ритма, сердечной недостаточностью и внезапной смертью $[1,2,3]$. Одним из факторов, способствующих снижению фатальных рисков у пациентов с острой коронарной патологией (ОКП), считается достижение целевых значений частоты сердечных сокращений (ЧСС) до 55-60 уд/мин. Установлено, что независимо от механизма, благодаря которому уменьшается ЧСС в покое, при ее уменьшении на 10 уд/мин относительный риск сердечной смерти сокращается на $30 \%$, риск внезапной смерти на $39 \%$, от- носительный риск смерти от всех причин - на $20 \%[9,10]$. Однако в реальной клинической практике далеко не у всех пациентов удается достичь рекомендованного уровня ЧСС, поскольку регулярность сердечного ритма находится под влиянием разнообразных факторов, таких как пол, возраст, характер нарушения вегетативной регуляции, эмоциональное состояние, температура окружающей среды и многие другие [7]. В этой связи представляется важным в дебюте госпитальной реабилитации выделить пациентов, достижение целевых значений ЧСС у которых потребует дополнительных усилий, связанных с подбором пульсурежающей терапии; такие пациенты нуждаются в особом внимании при самоконтроле и со стороны врача-кардиолога и реабилитолога. Особо перспективным представляется поиск параметров, ассоциирующихся с «недо- 
стижением» целевых уровней ЧСС на фоне традиционной терапии в период реабилитации больных ОКП, что позволит разработать ранние прогностические критерии достижения целевых значений ЧСС на госпитальных этапах у таких больных. Современные программно-аппаратные комплексы (ПАК) для оценки функционального состояния (ФС) организма позволяют на основе математического анализа ритма сердца получать информацию о регуляции сердечного ритма с четырех уровней его управления: периферического, вегетативного, гипоталамо-гипофизарного и центрального [6]. Учитывая, что значение ЧСС является результатом интеграции множества факторов, включающих адаптационные механизмы, вегетативную и центральную регуляцию, психоэмоциональный статус, важным для поиска прогностических критериев достижения целевых значений ЧСС является использование достаточно информативного метода комплексной оценки функционального состояния организма, учитывающего указанные факторы.

В этой связи целью настоящей работы стало исследование параметров функционального состояния пациентов с острой коронарной патологией в зависимости от достижения целевых значений ЧСС и определение показателей, взаимосвязанных со значением ЧСС на этапах госпитальной реабилитации.

\section{МАТЕРИАЛЫ И МЕТОДЫ ИССЛЕДОВАНИЯ}

Открытое рандомизированное исследование включало 197 пациентов (137 мужчин и 60 женщин), поступивших в отделение неотложной кардиологии с диагнозом «острый коронарный синдром» в возрасте 40-65 лет $(56,9 \pm 6,2)$. Ретроспективно с учетом окончательного диагноза пациенты были распределены на две группы: I - пациенты с НС (86 человек), II - пациенты с ОИМ (111 человек). В зависимости от величины чСС больные НС и ОИМ на каждом из этапов исследования распределялись на две подгруппы: a - пациенты с ЧСС 60 и менее уд/мин, б - пациенты с ЧСС 61 и более уд/мин. Вариационный размах ЧСС во время исследования был от 45 до 95 уд/мин.

Исследование было одобрено региональным этическим комитетом при ФГБОУ ВО «Курский государственный медицинский университет» Минздрава России (протокол № 9 от 09.11.15 г.).

Критерии включения в исследование: возраст, наличие ОКП, регулярный синусовый ритм во время исследований, информированное согласие. Критерии исключения: кардиогенный шок на момент включения, отказ от участия. Критерии рандомизации: пол, возраст.
Диагноз «инфаркт миокарда» и «нестабильная стенокардия» устанавливался согласно общепринятым критериям с учетом маркеров некроза миокарда (тропонин Т или I, MB-фракция креатинфосфокиназы), наличия типичной клинической картины и характерных изменений на ЭКГ [11].

Медикаментозная терапия соответствовала рекомендованным стандартам и включала: наркотические и ненаркотические анальгетики, антиагреганты и антикоагулянты, бета-блокаторы, ингибиторы АПФ или сартаны, статины, нитраты и диуретики при необходимости. Тромболитическая терапия была проведена 3 больным (1,5\% пациентов) с положительным эффектом, ангиопластика не проводилась в связи с наличием противопоказаний.

Клинико-анамнестическая характеристика исследуемых больных представлена в таблице 1 .

Всем пациентам проводилось исследование ФС организма с помощью ПАК «Омега-М» научно-производственной фирмы «Динамика» (г. Санкт Петербург, регистрационное удостоверение № ФСР 2010/09117 от 01.11.2010 г.) на 3-и, 8-е и 14-е сутки пребывания в стационаре.

В ПАК «Омега-М» присутствует алгоритмический блок цифрового анализа кардиоритмов. Специализированная компьютерная программа переводит ритмограмму в двоичную систему исчисления. Далее, анализируя двоичный код, выявляются уникальные последовательности (нейродинамические коды), соответствующие периодам колебаний регуляторных систем (вегетативной, нейрогуморальной, центральной). По свойствам нейродинамических кодов делалось заключение о характере и степени нарушений на каждом вышестоящем уровне регуляции сердечного ритма [8]. Подобный программный комплекс был апробирован для скрининг-диагностики внутренних заболеваний и оценки эффективности лечебно-профилактических мероприятий, доказав свою высокую диагностическую и экономическую эффективность в клинической практике [5].

В ходе работы оценивалась ЧСС и следующие интегральные показатели ФС организма пациентов НС и ОИМ:

А - показатель адаптации сердечнососудистой системы,

В - показатель вегетативной регуляции,

C - показатель центральной регуляции,

D - показатель психоэмоционального состояния $[4,5,6,8]$.

Оценка вышеуказанных параметров ФС представляется в \%, норма у здоровых лиц составляет 60-100\%, а референсные значения для пациентов с сердечно-сосудистыми заболеваниями в настоящий момент не разработаны $[4,5,6,8]$. 
Таблица 1

Клинико-анамнестическая характеристика исследуемых пациентов $(\mathrm{M} \pm \mathrm{m})$

\begin{tabular}{|l|c|c|c|}
\hline \multicolumn{1}{|c|}{ Показатель } & $\mathrm{HC}(\mathrm{n}=86)$ & $\mathrm{OИM} \mathrm{(n=111)}$ & Значение $\mathrm{p}$ \\
\hline Пол, муж/жен & $70,9 / 29,1(61 / 25)$ & $68,5 / 31,5(76 / 35)$ & 0,710 \\
\hline Возраст & $56,6 \pm 0,7$ & $57,2 \pm 0,6$ & 0,492 \\
\hline Постинфарктный кардиосклероз & $60,47 \%(52)$ & $21,62 \%(24)$ & $\mathrm{p}<0,001$ \\
\hline Отек легких & $1,16 \%(1)$ & $8,11 \%(9)$ & 0,013 \\
\hline Сердечная астма & $3,49 \%(3)$ & $1,80 \%(2)$ & 0,459 \\
\hline Кардиогенный шок & $0 \%(0)$ & $2,70 \%(3)$ & 0,023 \\
\hline Гипертонический криз & $2,33 \%(2)$ & $1,80 \%(2)$ & 0,795 \\
\hline Эпистенокардитический перикардит & $0 \%(0)$ & $0,90 \%(1)$ & 0,188 \\
\hline Последствия ОНМК & $9,30 \%(8)$ & $10,81 \%(12)$ & 0,727 \\
\hline Цереброваскулярная болезнь & $3,49 \%(3)$ & $11,71 \%(13)$ & 0,026 \\
\hline Сахарный диабет 2 типа & $17,44 \%(15)$ & $27,03 \%(30)$ & 0,108 \\
\hline Креатинин, ммоль/л & $103,77 \pm 3,50$ & $102,01 \pm 2,18$ & 0,669 \\
\hline Мочевина, ммоль/л & $6,23 \pm 0,24$ & $6,27 \pm 0,24$ & 0,899 \\
\hline Общий белок, г/л & $70,32 \pm 0,77$ & $69,28 \pm 1,04$ & 0,421 \\
\hline Общий холестерин, ммоль/л & $4,95 \pm 0,15$ & $5,02 \pm 0,14$ & 0,745 \\
\hline Триглицериды, моль/л & $1,72 \pm 0,17$ & $1,69 \pm 0,12$ & 0,890 \\
\hline ЛПВП, ммоль/л & $1,02 \pm 0,07$ & $0,95 \pm 0,06$ & 0,423 \\
\hline ЛПНП, ммоль/л & $3,12 \pm 0,39$ & $3,32 \pm 0,32$ & 0,689 \\
\hline Глюкоза, ммоль/л & $5,26 \pm 0,23$ & $4,52 \pm 0,21$ & 0,435 \\
\hline Общий билирубин, мкмоль/л & $12,86 \pm 0,52$ & $13,90 \pm 0,80$ & 0,280 \\
\hline
\end{tabular}

Примечание: ОНМК - острая недостаточность мозгового кровообращения, ЛПВП - липопротеиды высокой плотности, ЛПНП - липопротеиды низкой плотности.

Статистическая обработка результатов осуществлялась с помощью пакета программ «Омега-М» (ООО «Динамика», г. Санкт-Петербург) и пакета прикладных статистических программ SAS (Statistical Analysis System, SAS Institute, Cary, NC, USA). Результаты представлены в виде средних арифметических значений и их стандартной ошибки $(\mathrm{M} \pm \mathrm{m})$. Межгрупповое сравнение проводили с помощью критерия Стьюдента. Связь между показателями рассчитывалась по линейной корреляции Пирсона (r) и ранговой корреляции Спирмена (R), которые оценивались следующим образом: 0-0,25 - слабая, 0,25-0,5 средняя, 0,5-0,75 - сильная, 0,75-1 - очень сильная. Достоверными считались результаты при уровне $\mathrm{p}<0,05$.

\section{РЕЗУЛЬТАТЫ ИССЛЕДОВАНИЯ И ИХ ОБСУЖДЕНИЕ}

В группе Іа, на всех этапах исследования уровень адаптации сердечно-сосудистой системы (A), центральной регуляции (C) и психоэмоционального состояния (D) колебался в пределах $32-36 \%$ и не был подвержен динамическим изменениям на этапах госпитальной реабилитации, уровень вегетативной регуляции (В) на 3-и и 8 -е сут составил $54 \%$, а на 14 -е сут составил $59 \%$. Значения интегральных показателей ФС пациентов группы Іб находились в диапазоне $\approx 20-30 \%$, причем так же как и в группе Іа, наиболее высокие значения были у показателя В на всех этапах исследования, динамические изменения регистрировались в отношении показателя С, величина которого снизилась на 5\% к 8-м и 14-м сут. Различия между пациентами Іа и Іб группами на 3-и сут проявлялись в отношении значений показателя А и В, а на 8-е и 14-е сут - в отношении показателей $\mathrm{A}, \mathrm{B}, \mathrm{C}$ и $\mathrm{D}$ (табл. 2).

В группе ІІа на всех этапах исследования уровень показателей $\mathrm{A}, \mathrm{C}$ и $\mathrm{D}$ колебался в пределах 27-34\%, а показатель В - 52-53\%; обращает внимание снижение значений показателя А с 3-х сут к 14-м сут на 5\%. Исследуемые показатели ФС в группе больных ІІб на этапах госпитальной реабилитации имели следующие значения: показатель А - 12-15\%, В - 22-27\%, С - 14-18\% и D - $16-20 \%$, обращает внимание, что именно в группе ІІб, по сравнению с группами Іа, Іб и ІІа, значения параметров ФС были минимальными. На фоне госпитальной реабилитации в группе IIб было отмечено изменение показателя В, значения которого от 3-х к 14-м сут повысились на 5\%. На всех этапах исследования были установлены различия между группами пациентов ІІа и ІІб по показателям A, B, С и D (табл. 3). 
Таблица 2

Показатели функционального состояния организма больных нестабильной стенокардии $(\mathrm{M} \pm \mathrm{m})$

\begin{tabular}{|c|c|c|c|c|c|c|}
\hline Дата & Группа & ЧCC & $\mathrm{A}$ & $\mathrm{B}$ & $\mathrm{C}$ & $\mathrm{D}$ \\
\hline \multirow{2}{*}{3 сут } & Ia $(n=34)$ & $54,6 \pm 0,7$ & $35,3 \pm 3,8$ & $54,4 \pm 4,3$ & $33,3 \pm 3,8$ & $36,1 \pm 3,8$ \\
\hline & Іб $(\mathrm{n}=52)$ & $70,9 \pm 1,1$ & $20,6 \pm 3,2$ & $31,8 \pm 3,5$ & $26,1 \pm 3,0$ & $27,7 \pm 3,1$ \\
\hline \multicolumn{2}{|c|}{ Значение $\mathrm{p}$} & $<0,001$ & 0,004 & $<0,001$ & 0,136 & 0,091 \\
\hline \multirow{2}{*}{8 сут } & Ia $(n=44)$ & $55,3 \pm 0,6$ & $32,8 \pm 2,9$ & $54,0 \pm 3,6$ & $32,4 \pm 2,9$ & $34,9 \pm 2,8$ \\
\hline & Іб $(n=42)$ & $71,0 \pm 1,1$ & $19,6 \pm 4,1$ & $29,2 \pm 4,3$ & $21,6 \pm 3,7$ & $23,5 \pm 4,0$ \\
\hline \multicolumn{2}{|c|}{ Значение $\mathrm{p}$} & $<0,001$ & 0,010 & $<0,001$ & 0,023 & 0,022 \\
\hline \multirow{2}{*}{14 сут } & Ia $(n=38)$ & $55,1 \pm 0,7$ & $36,0 \pm 2,6$ & $59,3 \pm 3,8$ & $34,7 \pm 2,9$ & $35,5 \pm 2,7$ \\
\hline & Іб $(n=48)$ & $67,9 \pm 0,9$ & $20,3 \pm 4,3$ & $32,0 \pm 4,3$ & $21,3 \pm 3,6$ & $24,2 \pm 3,9$ \\
\hline \multicolumn{2}{|c|}{ Значение $\mathrm{p}$} & $<0,001$ & 0,003 & $<0,001$ & 0,005 & 0,019 \\
\hline
\end{tabular}

Примечание: Іа - группа пациентов НС с ЧСС 60 и менее ударов в минуту, Іб - группа пациентов НС с ЧСС 61 и более ударов в минуту.

Таблица 3

Показатели функционального состояния организма больных острым инфарктом миокарда $(\mathrm{M} \pm \mathrm{m})$

\begin{tabular}{|c|c|c|c|c|c|c|}
\hline Дата & Группа & ЧСС & A & B & C & D \\
\hline \multirow{2}{*}{3 сут } & IIa (n=41) & $55,7 \pm 0,5$ & $31,8 \pm 2,8$ & $53,4 \pm 3,9$ & $32,4 \pm 2,9$ & $33,7 \pm 3,0$ \\
\cline { 2 - 7 } & IIб (n=70) & $71,9 \pm 1,0$ & $11,6 \pm 1,6$ & $22,2 \pm 2,1$ & $14,0 \pm 1,5$ & $15,9 \pm 1,6$ \\
\hline \multicolumn{2}{|c|}{ Значение p } & $<0,001$ & 0,004 & $<0,001$ & $<0,001$ & $<0,001$ \\
\hline \multirow{2}{*}{8 сут } & IIa (n=41) & $54,9 \pm 0,6$ & $28,7 \pm 3,0$ & $53,0 \pm 4,0$ & $30,2 \pm 3,4$ & $33,8 \pm 3,4$ \\
\cline { 2 - 7 } & IIб (n=70) & $70,5 \pm 0,8$ & $12,3 \pm 1,8$ & $23,3 \pm 2,5$ & $16,9 \pm 2,1$ & $16,9 \pm 2,0$ \\
\hline \multicolumn{2}{|c|}{ 3начение p } & $<0,001$ & 0,010 & $<0,001$ & $<0,001$ & 0,001 \\
\hline \multirow{2}{*}{14 сут } & IIa (n=40) & $55,0 \pm 0,6$ & $26,6 \pm 2,9$ & $52,4 \pm 3,6$ & $28,8 \pm 3,0$ & $31,4 \pm 3,1$ \\
\cline { 2 - 7 } & IIб (n=71) & $68,3 \pm 0,7$ & $14,8 \pm 1,9$ & $26,8 \pm 2,6$ & $17,7 \pm 2,1$ & $20,1 \pm 2,2$ \\
\hline \multicolumn{2}{|c|}{ 3начение p } & $<0,001$ & 0,003 & 0,001 & $<0,001$ & 0,003 \\
\hline
\end{tabular}

Примечание: ІІа - группа пациентов ОИМ с ЧСС 60 и менее ударов в минуту, ІІб - группа пациентов ОИМ с ЧСС 61 и более ударов в минуту.

Полученные результаты свидетельствуют о том, что значения интегральных параметров ФС пациентов ОКП на всех этапах госпитальной реабилитации находились ниже нормы, однако в случае достижения целевых значений ЧСС как в группе больных НС, так в группе больных ОИМ значения показателей $\mathrm{A}, \mathrm{B}, \mathrm{C}$ и D были более высокими, особенно, уровень вегетативной регуляции (B).

При корреляционном анализе значений ЧСС и интегральных показателей ФС организма пациентов НС и ОИМ было установлено наличие обратной связи средней и сильной степени между ними (табл. 4).

Из таблицы 4 следует, что у пациентов с НС значения ЧСС на 3-и сут имели обратную корреляционную связь с показателями ФС на 3 -и сут $\mathrm{A}(-0,30)$ и $\mathrm{B}(-0,52)$, а также на 8 -е сут $\mathrm{B}(-0,35)$ и D $(-0,25)$. Между ЧСС на 8-е сут была установлена обратная связь средней силы с показателями А $(-0,29)$, В $(-0,47)$, C $(-0,26)$ и D $(-0,27)$ на 8-е сут и показателем В на 3-и сут $(-0,33)$. Наличие корреляционной связи средней силы установлено между ЧСС на 14-е сут и следующими показателями: на 3-и сут - B, на 8-е сут - A, B, C и D, на 14-е сут -
$\mathrm{A}, \mathrm{C}$ и $\mathrm{D}$, а сильная корреляционная связь была зарегистрирована между ЧСС на 14-е сут и показателем В на 14 -е сут $(-0,54)$. Таким образом, у пациентов НС с 3-х сут значения ЧСС взаимосвязаны с уровнем адаптации сердечно-сосудистой системы и уровнем вегетативной регуляции, а с 8-х еще и с уровнем центральной регуляции и психоэмоциональным состоянием, но стоит отметить, что с уровнем вегетативной регуляции связь наиболее сильная.

В группе ОИМ на протяжении всего периода наблюдения было установлено наличие корреляционной связи ЧСС с показателями ФС, однако, ее сила на этапах исследования была не одинакова. Примечательно, что сильная связь ЧСС на 3-и сут была с показателем В на 3-и $(-0,58)$ и 14-е $(-0,51)$ сут и показателем С на $3-и$ сут $(-0,51)$, в то время как на 8-е сут ЧСС имела сильную связь с показателями 8-х сут А $(-0,52)$, В $(-0,63)$ и $\mathrm{D}(-0,50)$ и $14-\mathrm{x}$ сут $\mathrm{B}(-0,53)$, а на $14-\mathrm{-} \mathrm{сут} \mathrm{силь-}$ ная связь ЧСС сохранялась только с показателем В 14-х сут. 
Таблица 4

Корреляционная связь (r) между ЧСС и интегральными показателями функционального состояния больных нестабильной стенокардией $(\mathrm{n}=86)$ и острым инфарктом миокарда $(\mathrm{n}=111)$

\begin{tabular}{|c|c|c|c|c|c|c|c|}
\hline \multirow{2}{*}{ Показатели } & \multirow{2}{*}{ Дата } & \multicolumn{3}{|c|}{ ЧСС в группе НС } & \multicolumn{3}{|c|}{ ЧСС в группе ОИМ } \\
\hline & & 3 сут & 8 cyт & 14 сут & 3 сут & 8 сут & 14 сут \\
\hline \multirow{3}{*}{ A } & 3 сут & $\begin{array}{c}-0,30 \\
p=0,005\end{array}$ & н/д & $\begin{array}{c}-0,23 \\
p=0,033\end{array}$ & $\begin{array}{c}-0,48 \\
p<0,001\end{array}$ & $\begin{array}{c}-0,42 \\
p<0,001\end{array}$ & $\begin{array}{c}-0,28 \\
\mathrm{P}=0,003\end{array}$ \\
\hline & 8 сут & $\begin{array}{c}-0,21 \\
\mathrm{p}=0,048\end{array}$ & $\begin{array}{c}-0,29 \\
\mathrm{p}=0,006\end{array}$ & $\begin{array}{c}-0,28 \\
\mathrm{p}=0,008\end{array}$ & $\begin{array}{c}-0,44 \\
p<0,001\end{array}$ & $\begin{array}{c}-0,52 \\
p<0,001\end{array}$ & $\begin{array}{c}-0,30 \\
p<0,001\end{array}$ \\
\hline & 14 сут & $\mathrm{H} /$ Д & $\mathrm{H} /$ Д & $\begin{array}{c}-0,38 \\
p<0,001\end{array}$ & $\begin{array}{c}-0,32 \\
p=0,003\end{array}$ & $\begin{array}{c}-0,33 \\
p=0,002\end{array}$ & $\begin{array}{c}-0,39 \\
\mathrm{p}<0,001\end{array}$ \\
\hline \multirow{3}{*}{ B } & 3 сут & $\begin{array}{c}-0,52 \\
p<0,001\end{array}$ & $\begin{array}{c}-0,33 \\
p=0,002\end{array}$ & $\begin{array}{c}-0,39 \\
p<0,001\end{array}$ & $\begin{array}{c}-0,58 \\
p<0,001\end{array}$ & $\begin{array}{c}-0,46 \\
p<0,001\end{array}$ & $\begin{array}{c}-0,33 \\
p<0,001\end{array}$ \\
\hline & 8 сут & $\begin{array}{c}-0,35 \\
\mathrm{p}<0,001\end{array}$ & $\begin{array}{c}-0,47 \\
\mathrm{p}<0,001\end{array}$ & $\begin{array}{c}-0,45 \\
\mathrm{p}<0,001\end{array}$ & $\begin{array}{c}-0,49 \\
\mathrm{p}<0,001\end{array}$ & $\begin{array}{c}-0,63 \\
\mathrm{p}<0,001\end{array}$ & $\begin{array}{c}-0,40 \\
\mathrm{p}<0,001\end{array}$ \\
\hline & 14 сут & $\mathrm{H} /$ д & $\mathrm{H} /$ д & $\begin{array}{c}-0,54 \\
p<0,001\end{array}$ & $\begin{array}{c}-0,51 \\
p<0,001\end{array}$ & $\begin{array}{c}-0,53 \\
p<0,001\end{array}$ & $\begin{array}{c}-0,56 \\
\mathrm{p}<0,001\end{array}$ \\
\hline \multirow{3}{*}{$\mathrm{C}$} & 3 сут & н/д & н/д & н/д & $\begin{array}{c}-0,51 \\
p<0,001\end{array}$ & $\begin{array}{c}-0,41 \\
p<0,001\end{array}$ & $\begin{array}{c}-0,23 \\
\mathrm{p}=0,014\end{array}$ \\
\hline & 8 сут & $\mathrm{H} /$ д & $\begin{array}{c}-0,26 \\
\mathrm{p}=0,014\end{array}$ & $\begin{array}{c}-0,29 \\
\mathrm{p}=0,007\end{array}$ & $\begin{array}{c}-0,42 \\
\mathrm{p}<0,001\end{array}$ & $\begin{array}{c}-0,44 \\
\mathrm{p}<0,001\end{array}$ & $\begin{array}{c}-0,23 \\
\mathrm{p}=0,013\end{array}$ \\
\hline & 14 сут & н/д & $\mathrm{H} /$ Д & $\begin{array}{c}-0,38 \\
p<0,001\end{array}$ & $\begin{array}{c}-0,35 \\
p<0,001\end{array}$ & $\begin{array}{c}-0,35 \\
p<0,001\end{array}$ & $\begin{array}{c}-0,32 \\
p<0,001\end{array}$ \\
\hline \multirow{3}{*}{ D } & 3 сут & $\begin{array}{c}-0,21 \\
p=0,045\end{array}$ & $\mathrm{H} /$ д & н/д & $\begin{array}{c}-0,47 \\
p<0,001\end{array}$ & $\begin{array}{c}-0,36 \\
p<0,001\end{array}$ & $\begin{array}{c}-0,23 \\
p=0,013\end{array}$ \\
\hline & 8 сут & $\begin{array}{c}-0,25 \\
p=0,021\end{array}$ & $\begin{array}{c}-0,27 \\
p=0,013\end{array}$ & $\begin{array}{c}-0,34 \\
p=0,002\end{array}$ & $\begin{array}{c}-0,44 \\
p<0,001\end{array}$ & $\begin{array}{c}-0,50 \\
p<0,001\end{array}$ & $\begin{array}{c}-0,26 \\
p=0,005\end{array}$ \\
\hline & 14 сут & н/д & н/д & $\begin{array}{c}-0,35 \\
p=0,001\end{array}$ & $\begin{array}{c}-0,35 \\
p<0,001\end{array}$ & $\begin{array}{c}-0,38 \\
p<0,001\end{array}$ & $\begin{array}{c}-0,33 \\
p<0,001\end{array}$ \\
\hline
\end{tabular}

Примечание: н/д - не достоверная связь, $\mathrm{p}>0,05$.

Таким образом, наличие взаимосвязи ЧСС на каждом из этапов исследования с показателями А, B, С и D на 3-и, 8-е и 14-е сут подтверждает влияние исследуемых составляющих ФС организма на формирование уровня ЧСС. Полученные результаты свидетельствуют о том, что наиболее тесная взаимосвязь имеет место между ЧСС и показателем В, что в дальнейшем может позволить использовать его в качестве прогностического критерия, определяющего уровень ЧСС на этапах госпитальной реабилитации.

Проведенное исследование позволило установить, что у пациентов с ОКП функциональное состояние организма на всех этапах госпитальной реабилитации находится ниже нормы, а уровень вегетативной регуляции выше, чем уровень адаптации сердечно-сосудистой системы, центральной регуляции и психоэмоционального состояния на протяжении всего госпитального периода.

Использование ПАК «Омега-М» позволяет проводить динамический контроль ФС пациентов ОКП по параметрам, отражающим различные уровни регуляции сердечного ритма, что может помочь врачу в выборе пульсурежающей терапии, выборе средств, направленных на улучшение психоэмоционального состояния и процессов центральной регуляции у таких пациентов.

Таким образом, на основании проведенных исследований можно сделать следующие выводы:

1. У больных ОКП в период госпитальной реабилитации значения параметров ФС, характеризующие адаптацию сердечно-сосудистой системы (A), вегетативную и центральную регуляции (В и C), психоэмоциональное состояние (D), при их оценке ПАК «Омега-М» находятся в корреляционной связи с величиной ЧСС, степень которой зависит от срока госпитальной реабилитации.

2. У больных как НС, так и ОИМ величина показателя вегетативной регуляции (В), оцененная на 3-и сут с момента развития заболевания, имеет корреляционную связь средней силы со значением ЧСС на 8-е и 14-е сут болезни, что позволяет использовать указанный параметр при прогностической оценке возможности достижения целевого уровня ЧСС в период госпитальной реабилитации. 


\section{ЛИТЕРАТУРА / REFERENCES}

1. Бокерия Л.А., Гудкова Р.Г. Болезни системы кровообращения и сердечно-сосудистая хирургия в Российской Федерации. Состояние и проблемы // Аналитический вестник Совета Федерации Федерального Собрания РФ. - 2015. - № 597 (44) C. 9-18. [Bokeriya L.A., Gudkova R.G. Diseases of the circulatory system and cardiovascular surgery in the Russian Federation. Status and problems. Analiticheskiy vestnik Soveta Federatsii Federal'nogo Sobraniya RF. 2015; 597 (44): 9-18 (in Russ.)].

2. Елисеева М.А., Курочкина О.Н. Течение острого инфаркта миокарда по данным ретроспективного исследования // Кардиология. - 2017. - Т. 57, № 5. - C. 30-32. [Eliseeva M.A., Kurochkina O.N. The Course of Acute Myocardial Infarction According to Date of a Retrospective Study. Kardiologiya. 2017; 57(5): 30-32 (in Russ.)].

3. Мариевич С.Ю., Гинзбург М.Л., Кутишенко Н.П., Загребальный А.В., Балашов И.С., Гарькина Л.Г. Динамика догоспитальной терапии больных с острым коронарным синдромом с 2005 по 2014 гг. по данным регистра ЛИС // Рациональная фармакотерапия в кардиологии. - 2014. - Т. 10, № 6. - C. 631-633. [Marcevich S.Ju., Ginzburg M.L., Kutishenko N.P., Zagrebel'nyj A.V., Balashov I.S., Gar'kina L.G. Dynamics of prehospital pharmacotherapy in patients with acute coronary syndrome in 2005-2014 according to the LIS register. Racional'naja farmakoterapija v kardiologii. 2014; 10 (6): 631-633 (in Russ.)].

4. Михин В.П., Коробова В.Н., Харченко А.В., Спасский А.А., Каюшников А.Б., Ледовский С.М., Маркина Т.Н., Чернятина М.A. Сравнительная оценка психоэмоционального и функционального состояния пациентов с острым коронарным синдромом в зависимости от его исхода // Курский научно-практический вестник «Человек и его здоровье». - 2018. - № 1. - C. 25-29. - DOI: 10.21626/vestnik/2018-1/04. [Mikhin V.P., Korobova V.N., Kharchenko A.V., Spasskiy A.A., Kayushnikov A.B., Ledovskiy S.M., Markina T.N., Chernyatina M.A. Comparative assessment of psycho-emotional and functional state of patients with acute coronary syndrome according to its outcome. Kurskiy nauchnoprakticheskiy vestnik "Chelovek i ego zdorov'ye". Kursk Scientific and Practical Bulletin "Man and His Health". 2018; (1): 25-29 (in Russ.)].

5. Обоснование аппаратно-программных методов, предназначенных для скрининг-диагностики внутренних заболеваний и для оценки эффективности лечебно-профилактических мероприятий в системе диспансеризации военнослужащих и пенсионеров МO: отчёт о научно-исслед. работе. - СПб : Изд-во ВМА, 2002. - 77 c. [Substantiation of the hardware and software methods intended for screening and diagnosis of internal diseases and for assessing the effectiveness of therapeutic and prophylactic measures in the system of medical examination of servicemen and retirees of the Ministry of Defense: a report on the scientific-research work.. - SPb : Izd-vo VMA; 2002: 77 (in Russ.)]

6. Смирнов К.Ю., Смирнов Ю.А. Разработка и исследование методов математического моделирования и анализа биоэлектрических сигналов. - СПб., 2001. - 43 с. [Smirnov K.Yu., Smirnov Yu.A. Development and research of methods of mathematical modeling and analysis of bioelectric signals. - SPb.; 2001: 43 (in Russ.)].

7. Умбрасиене Е., Вениловиене Й., Бабарскиене Р.М. Влияние частоты сердечных сокращений на смертность в течение одного года у больных ишемической болезнью сердца // Кардиология. 2011. - T. 51, № 10. - C. 15-20. [Umbrasiene J., Vencloviene J., Babarskiene R.M. Resting Heart Rate and One Year Mortality Association in Patients With Coronary Heart Disease. Kardiologiya. 2011; 51(10): 15-20 (in Russ.)].

8. Ярилов С.В. Физиологические аспекты новой информационной технологии анализа биоэлектрических сигналов и принципы технической реализации. - СПб, 2001. - 48 с. [Yarilov S.V. Physiological aspects of the new information technology for the analysis of bioelectric signals and the principles of technical implementation. - $\mathrm{SPb}$; 2001: 48 (in Russ.)].

9. Cooney M.T., Vartiainen E., Laatikainen T., Juolevi A., Dudina A., Graham I.M. Elevated Resting Heart Rate is an Independent Risk Factor for Cardiovascular Disease in Healthy Men and Women // Am Heart J. - 2010. - Vol. 159, N 4. - P. 612-619. DOI: 10.1016/j.ahj.2009.12.029.

10. Cucherat M. Quantitative relationship between resting heart rate reduction and magnitude of clinical benefits in post-myocardial infarction: a meta-regression of randomized clinical trials // Eur Heart J. - 2007. Vol. 28, N 24. - P. 3012-3019. - DOI: 10.1093/eurheartj/ehm489

11. Hamm C.W., Bassand J.P., Agewall S., Bax J., Boersma E., Bueno H., Caso P., Dudek D., Gielen S., Huber K., Ohman M., Petrie M.C., Sonntag F., Uva M.S., Storey R.F., Wijns W., Zahger D.; European Society of Cardiology. [ESC guidelines for the management of acute coronary syndromes in patients presenting without persistent ST-segment elevation. The Task Force for the management of acute coronary syndromes (ACS) in patients presenting without persistent ST-segment elevation of the European Society of Cardiology (ESC)] // G Ital Cardiol (Rome). - 2012. - Vol. 13, N 3. - P. 171228. - DOI: 10.1714/1038.11322. 